\title{
HIV medication adherence, substance use, sexual risk behaviors and psychological distress among younger black men who have sex with men and transgender women: Preliminary findings
}

\author{
Dexter Voisin ${ }^{1}$, Timothy Walsh ${ }^{2}$, Natasha Flatt ${ }^{2}$, Rebecca Eavou ${ }^{2}$, Clara Bertozzi-Villa ${ }^{2}$, Milton \\ Eder $^{3}$, John Schneider ${ }^{1}$
}

1. School of Social Service Administration, University of Chicago, United States. 2. Department of Medicine and Health Studies, University of Chicago, United States. 3. Access Community Health Care Network, United States.

Correspondence: Dexter R. Voisin. Address: School of Social Service Administration, University of Chicago, United States. Email: d-voisin@uchicago.edu

Received: May 7, 2014

DOI : $10.5430 /$ jnep.v4n12p27

\author{
Accepted: September 2, 2014 Online Published: September 24, 2014
}

URL: http://dx.doi.org/10.5430/jnep.v4n12p27

\begin{abstract}
Background: In the United Sates, young Black men who have sex with men and transgender women (YBMSM/TW) ages 16 to 29 bear the highest burden of new HIV infections. HIV medication adherence is critical for maintaining the quality of life for infected persons, supporting secondary prevention efforts and reducing community viral loads. However, few studies have examined the relationship between medication adherence and health related factors such as substance use, sexual risk behaviors and psychological distress symptoms among YBMSM/TW. This represents the primary focus of this exploratory study.
\end{abstract}

Methods: Baseline data is from Project nGage, a RCT that enrolled 86 newly diagnosed YBMSM/TW (ages 18-29). Measures on medication adherence, substance use, sexual risks behaviors and psychological distress symptoms were assessed.

Results: Study findings indicated that medication adherence was related to less alcohol use, alcohol desire, alcohol compulsion, and having a partner who used marijuana as a sex drug. Findings also indicated that medication adherence was related to decreased feelings of psychological distress. Study findings did not indicate any relationship between medication adherence and group or unprotected anal sex.

Conclusion: Our study findings indicated that HIV medication adherence, among YBMSM/TW was a significant correlate of lower substance use and psychological distress, and that service providers may enhance such gains by promoting medication adherence.

\section{Key words}

HIV medication adherence, Young Black men who have sex with men and transgender women, Health factors 


\section{Introduction}

Young Black men who have sex with men and transgender women (YBMSM/TW) bear the highest burden of new human immunodeficiency virus (HIV) infections when compared to other men who have sex with men (MSM) populations ${ }^{[1]}$. In Chicago, YBMSM have new HIV infection rates that are 7 times higher than their White MSM counterparts ${ }^{[2]}$. Furthermore, TW are 48 times more likely to have an HIV diagnosis when compared to other adults, with a prevalence of $19.1 \%$ in a recent systematic review of 15 countries ${ }^{[3]}$. Given the disproportionate rates of new infections among this population, HIV medication adherence is critical to maintaining the life quality for infected persons, sustaining suppressed viral loads and promoting secondary community prevention efforts ${ }^{[4-6]}$. Preliminary findings from the first two years of the PARTNER study, in which no HIV infected individual with an undetectable viral load transmitted the virus to their partner, has established medication adherence as a critical tool toward the elimination of new HIV transmission events ${ }^{[7]}$.

Given the importance of medication adherence, several studies have examined its relationship to substance use and sexual risk behaviors. However, findings have been mixed or limited which suggests that more studies on medication adherence are warranted. For instance, two studies comprised of large multiethnic samples of heterosexual and gay adults indicated that HIV medication adherence was negatively correlated with unprotected anal or vaginal sex ${ }^{[8,9]}$. Unfortunately, these findings did not examine interaction effects to understand if the association is moderated by ethnicity and/or sexual orientation and it remains unclear whether this relationship was significant for YBMSM. In contrast, several studies that compare risk behavior before and after 1996 have reported that the availability of highly active antiretroviral therapy (HAART) was associated with increased risky sexual behaviors (e.g., unprotected anal sex; having multiple sexual partners; unprotected anal sex with two or more partners of unknown HIV serostatus) among MSM populations ${ }^{\text {[10-13] }}$. Additionally, given the propensity for higher levels of psychological distress associated with having an HIV diagnosis $^{[14,15]}$, it is unclear whether medication adherence might be related to less psychological distress. Therefore, given the above gaps in the extant literature and the lack of prior studies focused exclusively on YBMSM/TW who have an elevated risk for contracting HIV, more research is warranted to explain the relationship between medication adherence and health-related factors such as substance use, sexual risks and psychological distress.

Asset theory ${ }^{[16]}$ as it relates to substance and sexual risk behaviors, posits that individuals who have an increased belief that their future holds the promise of success might reduce engagement in substance and sexual and other risk behaviors. Consequently, we posited that YBMSM/TW who have an investment in their health, as operationalized by medication adherence, would be more likely to avoid negative substance and sexual risk behaviors and report less psychological distress. Asset theory is consistent with other behavioral and psychosocial theories (e.g., theory of reasoned action) ${ }^{[17]}$ which support the belief that having personal capital is related to more protective self-behaviors and positive behavioral outcomes.

\section{Methods}

Between October 2012 and January 2014 baseline data were collected from a randomized control trial (RCT) called Project nGage. The study design of Project nGage is described extensively elsewhere ${ }^{[18]}$. Briefly, this RCT explores the role of social support in improving HIV primary care for YBMSM/TW. Patients that have been successfully linked to care are randomized to an experimental arm consisting of enhanced social support or a control arm comprising of treatment as usual. Recruitment occurred at two study sites, one is based in a university hospital and the other is a federally qualified health center hosting university physicians. After three clinic visits following an HIV diagnosis, patients meeting the eligibility criteria were introduced to project staff to learn about Project nGage. Patients were eligible for study participation if they were biologically male, self-identified as African American or Black, were between the ages of 16 to 29, had a positive HIV diagnosis for greater than three months, and had disclosed their diagnosis to at least one person in their close social network. Transgender women were defined as participants that self-identify as transgender women. For transgender women in our study, the participant's assigned sex did not match their gender expression. Data were collected 
on laptop computers by research assistants during interviews to record participant responses. Participants were paid $\$ 25.00$ for completing the baseline survey. Institutional Review Board at the University of Chicago approved all study procedures.

\subsection{Demographics}

Participants reported their age and sexual orientation as shown in Table 1. Education was assessed by one item, "What is your highest level of schooling or highest degree, certificate, or license?" Response categories were $1=$ less than high school; 2 = high school/general equivalency diploma (GED); 3 = some college, 2-year college degree; 4-year college degree; 4 = master's degree; or 5 = doctoral degree, or professional degree (MD, JD).

\subsection{Medication adherence}

Medication adherence was measured by two items. First, participants were asked "Are you currently on meds?” Participants that reported no current HIV medications were asked why they were not on HIV medications. Response categories included “Not yet prescribed; meds prescribed, but haven't picked up yet; meds prescribed, but don't want to take them; meds prescribed, but haven't been into clinic; or other." Participants that reported currently being on HIV medication were asked "What percent from 0 to 100 did you take your medication as prescribed in the last 30 days? Zero percent time would mean 'none of the time', 50\% time indicated 'half of the time', and 100\% indicated 'all of the time' ${ }^{\text {[19] }}$. This adherence measure has been shown to be strongly correlated with HIV-1 viral load measurements in previous research ${ }^{[20]}$.

\subsection{Psychological distress}

Psychological distress was assessed from the Brief Symptom Inventory-18 (BSI-18) scale ${ }^{[21]}$. The BSI-18 is a condensed version of the BSI and can be used to screen for psychological distress at baseline during the past seven days. The BSI-18 includes 18 items and assesses Global Severity Index (GSI), a cumulative measure of psychological distress. Each item is rated on a 5-point Likert scale ranging from 0 "not at all” to 4 "extremely." Previous studies have found the BSI-18 to have adequate reliability and validity when compared to the longer version ${ }^{[22,23]}$. Previous research favors the use of the GSI as an indicator of overall psychological distress from the BSI-18, instead of the use of individual subscales ${ }^{[22]}$.

The GSI score was used as the measure of psychological distress in our study. The raw GSI score was computed by taking the mean of all item scores. Raw scores were converted to T-scores and a participant was considered to experience psychological distress if $\mathrm{T}>62$ according to the BSI-18 scoring instructions ${ }^{[24]}$. The psychological distress variable was transformed into a dichotomous variable with a T-score of 62 as the cut-off point.

\subsection{Substance use}

Alcohol frequency was assessed by a single item ${ }^{[25]}$ and has been shown to be a reliable and valid measure of substance use ${ }^{[22]}$. "During the past 3 months, how often have you used alcoholic beverages?" Response categories were ranked on a 5 -point Likert scale 1 "never” to 5 "daily or almost daily.”

Alcohol desire was assessed by a single item, "During the past 3 months, how often have you had a strong desire or urge to use alcoholic beverages?” Response categories were ranked on a 5-point Likert scale ranging from 1 "never” to 5 "daily or almost daily."

Alcohol concern was assessed by a single item, "Has a friend or relative or anyone else ever expressed concern about your use of alcohol?” (no/yes).

Alcohol compulsion was assessed by one item, "Have you ever tried and failed to control, cut down, or stop using alcohol?" (no/yes). 


\subsection{Sexual risks}

Marijuana as a sex drug was assessed by a single item "Have you ever used marijuana as a 'sex drug', that is to make sex easier, better, last longer, or something similar?” (no/yes).

Marijuana as a sex drug by a partner was assessed by two items. First, respondents were asked "As far as you know, have any of your sexual partners ever used any 'sex drugs'?” (no/yes). If the sexual partners had used sex drugs, the respondent was asked to specify the drugs their sex partners had used as sex drugs.

Unprotected anal sex was assessed by a single survey item, "Have you had unprotected anal sex with a male partner of unknown or different HIV-status in the past six months?” (no/yes).

Group sex was assessed by the item, “Have you participated in group sex in the past six months?” Group sex was defined as having sex with 2 or more persons at the same time (no/yes).

\subsection{Statistical analyses}

Univariate analyses were used to describe the overall sample. As earlier indicated, raw scores for each relevant measure were converted to T-scores. Next, individual logistic regression models examined the relationships between HIV medication adherence and health-related factors. Finally, we calculated prevalence ratios, their $95 \%$ confidence intervals, and respective $p$-values controlling for age based on prior empirical considerations ${ }^{[26,27]}$. Given our sample size and the exploratory nature of this study, we calculated $p<.10$. All data were analyzed using STATA version $12^{[28]}$.

\section{Results}

The analytic sample comprised of 86 YBMSM/TW ages 18 to 29 (SD = 3.06); two participants identified as transgender women. The majority of participants reported having more than a high school degree $(50 \%, n=43)$. T-scores for psychological distress ranged from 38.66 to 77.92 with cronbach alpha $=0.69$. Psychological distress was experienced by 10 (11.1\%) participants ( $\mathrm{T}>62)$. Mean scores for all continuous study variables are presented in Table 1.

Table 1. Characteristics of the Sample $(\mathrm{N}=86)$

\begin{tabular}{|c|c|c|c|c|c|c|}
\hline \multirow{2}{*}{ Variables } & \multicolumn{3}{|c|}{ MSM (N = 84) } & \multicolumn{3}{|c|}{ TW $(\mathbf{N}=2)$} \\
\hline & $\mathbf{N}(\%)$ & Mean & SD & $\mathbf{N}(\%)$ & Mean & SD \\
\hline \multicolumn{7}{|l|}{ Demographics } \\
\hline Age & & 23.7 & 3.06 & & 24.0 & 1.41 \\
\hline \multicolumn{7}{|l|}{ Education } \\
\hline Less than HS & $19(22.6)$ & & & $0(0.00)$ & & \\
\hline HS diploma & $23(27.4)$ & & & $1(50.0)$ & & \\
\hline Greater than HS & $42(50.0)$ & & & $1(50.0)$ & & \\
\hline Psychological Distress & $10(11.9)$ & & & $0(0.00)$ & & \\
\hline \multicolumn{7}{|l|}{ Substance Use } \\
\hline Alcohol frequency & & 3.05 & 0.99 & & 4.00 & 0.00 \\
\hline Alcohol desire & & 2.08 & 1.33 & & 2.00 & 1.41 \\
\hline Alcohol concern & $23(29.1)$ & & & $1(50.0)$ & & \\
\hline Alcohol compulsion & $23(29.1)$ & & & $1(50.0)$ & & \\
\hline \multicolumn{7}{|l|}{ Sex Risks } \\
\hline Marijuana as a sex drug & $19(22.6)$ & & & $2(100)$ & & \\
\hline Marijuana as partner sex drug & $19(22.6)$ & & & $2(100)$ & & \\
\hline Group sex & $9(10.7)$ & & & $0(0.00)$ & & \\
\hline Unprotected anal sex & $19(22.6)$ & & & $1(50.0)$ & & \\
\hline \multicolumn{7}{|l|}{ Medication Adherence } \\
\hline Current HIV medication & $75(89.3)$ & & & $0(0.00)$ & & \\
\hline Adherence (last 30 days) ${ }^{*}$ & & 87.8 & 21.5 & & & \\
\hline
\end{tabular}

*Among those who reported currently being on HIV medication; MSM: men who have sex with men; TW: transgender women. 
Current HIV medication use was reported by 75 participants in the last 30 days; nine MSM and two TW participants were not currently taking HIV medication. Of nine MSM not on HIV medication, three had not yet been prescribed HIV medication, five had been prescribed medication but had not picked up the prescription, and one had HIV medication prescribed but did not want to take medication. Neither TW had been prescribed HIV medication on account of a recent diagnosis. Based on a skewed distribution for the medication adherence variable, this variable was dichotomized into high/low based on the median split. Medication adherence ranged from 0 to $100 \%$ with a median of $95 \%$. Based on the median-split, 37 participants were classified into the "low" category (0-95\%) and 38 participants were classified into the “high” category (>95\%).

\section{Medication adherence and health-related factors}

Controlling for the effects of age, HIV medication adherence was negatively related to feelings of psychological distress. In addition, medication adherence was negatively associated with alcohol use, alcohol desire, alcohol compulsion, and having a partner who used marijuana as a sex drug. Study findings did not indicate any relationship between medication adherence and group or unprotected anal sex (see Table 2).

Table 2. Relationships Between Medication Adherence and Health-Related Factors of YBMSM/TW

\begin{tabular}{llll}
\hline Logistic Regression & AOR $^{\#}$ & 95\% CI & P-Value \\
\hline Psychological distress & 0.217 & $(0.043-1.101)$ & .065 \\
Marijuana as sex drug & 0.388 & $(0.120-1.260)$ & .120 \\
Marijuana as partner sex drug & 0.260 & $(0.074-0.893)$ & .033 \\
Group sex & 4.063 & $(0.730-22.60)$ & .109 \\
Unprotected anal sex & 0.625 & $(0.207-1.891)$ & .454 \\
Alcohol frequency & 0.577 & $(0.338-0.984)$ & .044 \\
Alcohol desire & 0.715 & $(0.487-1.051)$ & .087 \\
Alcohol concern & 0.433 & $(0.146-1.275)$ & .130 \\
Alcohol compulsion & 0.201 & $(0.040-1.008)$ & .051 \\
\hline
\end{tabular}

\# All models controlled for the effects of age

\section{Discussion}

Despite the significant burden that YBMSM/TW experience with regards to new HIV infections ${ }^{[29]}$, few studies have focused on the relationship between HIV medication adherence and health related factors among this population. An understanding of medication adherence and its relationship to health-related factors is crucial to the development of interventions that integrate behavioral and biomedical domains ${ }^{[30]}$. This study makes an important empirical contribution by documenting that medication adherence is correlated with decreased psychological distress, a broad spectrum of alcohol risk behaviors and having a partner who used marijuana as a sex drug. Consequently, these results corroborate earlier findings based on multiethnic samples of heterosexual and gay adults indicating the adherence was associated with decreased substance use ${ }^{[31]}$. It also extends these findings by indicating that HIV adherence is associated with decreased psychological distress which is a significant mediator of sexual risk taking behavior ${ }^{[2,33}{ }^{33}$. Study findings did not document a correlation between UAS or group sex. Earlier studies have reported mixed findings and documented that medication adherence ${ }^{[8]}$ and availability ${ }^{[10-13]}$ are related to lower and higher UAS, respectively. Clearly larger sample sizes might be needed to provide more precise estimates and clarify this relationship.

Our hypothesis, grounded in asset theory, was confirmed by the significant relationship between medication adherence and a number of health-related factors. Demonstrating a greater investment in one's own health through medication adherence was found to be related to lower substance use and psychological distress. 
Several study limitations warrant mentioning. The cross sectional design does not allow us to disentangle temporal ordering among variables. For example, lower levels of psychological distress might lead to higher levels of medication adherence and vice-versa, and all relationships noted in this study are correlational with no causal inferences. In addition, though commonly used in social science research, all self-reported data has inherent limitations, and sample selection bias is possible given that a significant number of participants were recruited from a federally qualified health center. Our selection criteria included all persons who were born biologically male and therefore two TW were included in the study. We included them in the analytic sample given that they are often excluded from research studies. However, this population faces unique challenges and larger samples stratified by MSM and TW would be needed to yield precise estimates of the relationship between medication adherence and the health-related factors assessed in this study. Finally, given our sample size and the exploratory nature of this study, use of the median-split to create a low and high group for medication adherence may have contributed to a loss in power as described by Cohen ${ }^{[34]}$. Yet, dichotomization in our study was necessary to accommodate a wide range of participant responses for this measure. Despite these limitations, this exploratory study describes an important association between medication adherence and substance use, psychological distress, and partner drug use during sex. Consequently, clinical and behavioral staff might educate patients that medication adherence is correlated with lower psychological distress and decreased substance use indicators. In addition, future research with larger sample sizes would need to illuminate the pathways through which medication adherence is related to lower psychological distress and substance use. Consequently, psychoeducational interventions focused on promoting and reducing the barriers to medication adherence, might be correlated with important health-related factors for this highly vulnerable population.

\section{References}

[1] Johnson, A.S., et al. HIV Infection-United States, 2008 and 2010. CDC Health Disparities and Inequalities Report-United States. 2013; 62(3): 112.

[2] CDPH, H. Prevalence and Unrecognized Infection among Men Who Have Sex with Men-Chicago. Chicago Department of Public Health, 2008.

[3] Baral, S.D., et al. Worldwide burden of HIV in transgender women: a systematic review and meta-analysis. Lancet Infect Dis. 2013; 13(3): 214-22. http://dx.doi.org/10.1016/S1473-3099(12)70315-8

[4] Das, M., et al. Decreases in community viral load are accompanied by reductions in new HIV infections in San Francisco. PLoS One. 2010; 5(6): e11068. PMid:20548786 http://dx.doi.org/10.1371/journal.pone.0011068

[5] Montaner, J.S., et al. Association of highly active antiretroviral therapy coverage, population viral load, and yearly new HIV diagnoses in British Columbia, Canada: a population-based study. Lancet. 2010; 376(9740): 532-9. http://dx.doi.org/10.1016/S0140-6736(10)60936-1

[6] Porco, T.C., et al. Decline in HIV infectivity following the introduction of highly active antiretroviral therapy. Aids. 2004; 18(1): 81-8. PMid:15090833 http://dx.doi.org/10.1097/00002030-200401020-00010

[7] Rodger, A. HIV transmission risk through condomless sex if HIV+ partner on suppressive ART: PARTNER study, in 21st Conference on Retroviruses and Opportunistic Infections. 2014: Boston.

[8] Diamond, C., et al. Use of and adherence to antiretroviral therapy is associated with decreased sexual risk behavior in HIV clinic patients. J Acquir Immune Defic Syndr. 2005; 39(2): 211-8. PMid:15905739

[9] Remien, R.H., et al. Medication adherence and sexual risk behavior among HIV-infected adults: implications for transmission of resistant virus. AIDS Behav. 2007; 11(5): 663-75. PMid:17243012 http://dx.doi.org/10.1007/s10461-006-9201-8

[10] Chen, S.Y., et al. Continuing increases in sexual risk behavior and sexually transmitted diseases among men who have sex with men: San Francisco, Calif, 1999-2001, USA. Am J Public Health. 2002; 92(9): 1387-8. PMid:12197957 http://dx.doi.org/10.2105/AJPH.92.9.1387-a

[11] Dukers, N.H., et al. Sexual risk behaviour relates to the virological and immunological improvements during highly active antiretroviral therapy in HIV-1 infection. Aids. 2001; 15(3): 369-78. PMid:11273217 http://dx.doi.org/10.1097/00002030-200102160-00010

[12] Katz, M.H., et al. Impact of highly active antiretroviral treatment on HIV seroincidence among men who have sex with men: San Francisco. Am J Public Health. 2002; 92(3): 388-94. PMid:11867317 http://dx.doi.org/10.2105/AJPH.92.3.388 
[13] Scheer, S., et al. Effect of highly active antiretroviral therapy on diagnoses of sexually transmitted diseases in people with AIDS. Lancet. 2001; 357(9254): 432-5. http://dx.doi.org/10.1016/S0140-6736(00)04007-1

[14] Anderson, M., et al. "It took a piece of me": initial responses to a positive HIV diagnosis by Caribbean people in the UK. AIDS Care. 2010; 22(12): 1493-8. PMid:20824553 http://dx.doi.org/10.1080/09540121.2010.482125

[15] Perdices, M., et al. Anxiety, depression and HIV related symptomatology across the spectrum of HIV disease. Aust N Z J Psychiatry. 1992; 26(4): 560-6. PMid:1476521 http://dx.doi.org/10.3109/00048679209072089

[16] Sherraden, M. Stake holding: Notes on a theory of welfare based on assets. The Social Service Review. $1990 ; 580-601$. http://dx.doi.org/10.1086/603797

[17] Ajzen, I. and M. Fishbein. Understanding attitudes and predicting social behaviour. 1980.

[18] Bouris, A., et al. Project nGage: Network Supported HIV Care Engagement for Younger Black Men Who Have Sex with Men and Transgender Persons. J AIDS Clin Res. 2013; 4.

[19] Giordano, T.P., et al. Measuring adherence to antiretroviral therapy in a diverse population using a visual analogue scale. HIV Clin Trials. 2004; 5(2): 74-9. PMid:15116282 http://dx.doi.org/10.1310/JFXH-G3X2-EYM6-D6UG

[20] Naar-King, S., et al. Psychosocial factors and medication adherence in HIV-positive youth. AIDS Patient Care STDS. 2006; 20(1): 44-7. PMid:16426155 http://dx.doi.org/10.1089/apc.2006.20.44

[21] Derogatis, L.R. and P. Spencer. Brief Symptom Inventory: BSI. 1993: Pearson Upper Saddle River, NJ.

[22] Zabora, J., et al. A new psychosocial screening instrument for use with cancer patients. Psychosomatics. 2001; 42(3): 241-6. PMid:11351113 http://dx.doi.org/10.1176/appi.psy.42.3.241

[23] Andreu, Y., et al. Psychometric properties of the Brief Symptoms Inventory-18 (Bsi-18) in a Spanish sample of outpatients with psychiatric disorders. Psicothema. 2008; 20(4): 844-50. PMid:18940093

[24] Derogatis, L.R., BSI 18, Brief Symptom Inventory 18: Administration, scoring and procedures manual. 2001: NCS Pearson, Incorporated.

[25] Group, W. The alcohol, smoking and substance involvement screening test (ASSIST): development, reliability and feasibility. Addiction. 2002; 97(9): 1183-1194. http://dx.doi.org/10.1046/j.1360-0443.2002.00185.x

[26] Hinkin, C.H., et al. Medication adherence in HIV-infected adults: effect of patient age, cognitive status, and substance abuse. Aids. 2004; 18 Suppl 1: S19-25. PMid:15075494 http://dx.doi.org/10.1097/00002030-200401001-00004

[27] Barclay, T.R., et al. Age-associated predictors of medication adherence in HIV-positive adults: health beliefs, self-efficacy, and neurocognitive status. Health Psychol. 2007; 26(1): 40-9. PMid:17209696 http://dx.doi.org/10.1037/0278-6133.26.1.40

[28] StataCorp, Stata Statistical Software: Release 12. 2012, StataCorp LP: College Station, TX.

[29] Prejean, J., et al. Estimated HIV incidence in the United States, 2006-2009. PloS one. 2011; 6(8): e17502. PMid:21826193 http://dx.doi.org/10.1371/journal.pone.0017502

[30] Maulsby, C., et al. A systematic review of HIV interventions for black men who have sex with men (MSM). BMC Public Health. 2013; 13: 625. PMid:23819660 http://dx.doi.org/10.1186/1471-2458-13-625

[31] Hinkin, C.H., et al. Drug use and medication adherence among HIV-1 infected individuals. AIDS and Behavior, 2007. 11(2): p. 185-194. PMid:16897351 http://dx.doi.org/10.1007/s10461-006-9152-0

[32] Benotsch, E.G., S.C. Kalichman, and J.A. Kelly. Sexual compulsivity and substance use in HIV-seropositive men who have sex with men: prevalence and predictors of high-risk behaviors. Addict Behav. 1999; 24(6): 857-68. http://dx.doi.org/10.1016/S0306-4603(99)00056-8

[33] Gerbi, G.B., et al. A quantitative risk assessment of multiple factors influencing HIV/AIDS transmission through unprotected sex among HIV-seropositive men. AIDS Care. 2012; 24(3): 331-9. PMid:21895567 http://dx.doi.org/10.1080/09540121.2011.608418

[34] Cohen, J. The cost of dichotomization. Applied Psychological Measurement. 1983; 7: 249-253. http://dx.doi.org/10.1177/014662168300700301 